\title{
Violencia de género y prevención: una propuesta de lege ferenda realizada por el alumnado
}

Gender violence and prevention: a lege ferenda proposal made by students

DOI http://dx.doi.org/10.1344/REYD2018.18.24128

\section{Fernando Centenera Sánchez-Seco}

Profesor de Filosofía del Derecho. Departamento de Ciencias Jurídicas.

Universidad de Alcalá

E-mail: fernando.centenera@uah.es

Resumen. La violencia de género sigue siendo un problema importante en nuestros días. Esta preocupante situación, acentuada por las noticias que informan de repuntes, justifican sobradamente su consideración en el aula, tanto con vistas a descubrir su origen y sus manifestaciones, como en lo que respecta a la reflexión sobre posibles soluciones desde el punto de vista normativo. En este trabajo presentamos una experiencia docente desarrollada en la línea expuesta, que centra la atención en la prevención, y que se enmarca en una asignatura dedicada a la redacción de textos legales; novedosa en nuestro sistema docente, puesto que transmite competencias básicas para redactar normas. Concretamente, la experiencia consiste en la redacción individual por parte del alumnado, de una norma que modifica algunos aspectos de la actual ley estatal de medidas contra la violencia de género, en materia de prevención. Para ello, seguimos lo establecido en las Directrices de técnica normativa oficiales, en un desarrollo metodológico que contempla el título, la parte expositiva, la parte dispositiva y la parte final de la norma. El trabajo concluye con un apartado dedicado a la valoración de la experiencia, donde analizamos la opinión del alumnado sobre la misma y los resultados obtenidos, que confirman la importancia de formar en cuestiones de género, para finalmente dar paso a algunos aspectos planteados desde un punto de vista prospectivo.

Palabras clave: violencia de género, redacción legislativa, docencia. 
Abstract. Today, gender violence is still a major problem. This state of affairs, accentuated by news of upturns, is so alarming that its treatment in class, whether with a view to ascertaining its origin and manifestations or to pondering possible legal solutions, is amply justified. This paper presents a teaching experience carried out in this direction. Focusing on prevention, it forms part of a subject devoted to drafting legal texts which is a pioneer in our teaching system since it transmits basic competences for writing regulation. To be more precise, the experience required each student to draft a regulation modifying certain aspects of the current national anti-gender violence measures act. To this end, the students followed the indications of the official Technical Guidelines for Regulators, applying a structure consisting of the title, the exposition, the provisions, and the final part of the law. The paper concludes with a section appraising the experience, in which we analyses the students' opinions of the experience and the results obtained. These confirm the importance of providing training in gender-related issues. Finally, some suggestions are made for further work in this direction.

Keywords: gender violence, legal drafting, teaching. 


\title{
Violencia de género y prevención: una propuesta de lege ferenda realizada por el alumnado
}

\author{
Gender violence and prevention: a lege ferenda proposal made by students
}

\section{Introducción}

Desde hace ya varios años, en la Facultad de Derecho de la Universidad de Alcalá venimos ofertando una asignatura transversal dedicada a la Redacción de textos legales (2017-2018). En ella formamos al alumnado en la redacción de textos jurídicos, centrándonos en las normas, aunque no exclusivamente. Sobre el desarrollo de las prácticas que planteamos en la asignatura ya publicamos anteriormente un trabajo (CENTENERA SÁNCHEZ-SECO, 2016a). En él nos referimos a los ejercicios que realizamos sobre documentos administrativos, a los dedicados a cuestiones lingüísticas de tipo general y específico (siguiendo en este último ámbito las Directrices de técnica normativa de 2005), así como a la metodología que seguimos en términos generales, a la hora de elaborar el trabajo de fin de curso. Este último consiste en redactar de forma individual el texto de una norma que trate de afrontar un problema determinado.

En estas páginas presentamos un desarrollo concreto, enmarcado en el último ámbito citado, aunque también consideraremos de forma puntual algunos ejercicios sobre documentos administrativos. Concretamente, y aquí reside la originalidad de este artículo, nos referiremos a una experiencia llevada a cabo con el trabajo de fin de curso elaborado en el año académico recientemente finalizado. El ejercicio en cuestión ha consistido en redactar una norma que modifique la actual ley orgánica estatal sobre medidas contra la violencia de género, en materia de prevención. La persistencia del problema en la actualidad, que además viene acompañado de preocupantes repuntes, y la solicitud de la ley estatal citada para llevar a cabo desarrollos docentes en la línea que nos ocupa, han sido algunas de las motivaciones más relevantes que nos han invitado a trabajar sobre el tema en cuestión ${ }^{1}$. Debemos señalar además que las actividades que vamos a considerar se enmarcan en el siguiente proyecto de innovación docente de la Universidad de Alcalá: Avanzando en la visibilización de las mujeres y en soluciones normativas contra la violencia de género: algunas propuestas en el ámbito jurídico (UAH/EV911).

Para llevar a cabo el desarrollo anunciado hemos seguido el siguiente esquema ${ }^{2}$. En primer lugar, exponemos el diseño de la experiencia, haciendo especial referencia a los

\footnotetext{
${ }^{1}$ Hemos de señalar que por nuestra parte tuvimos oportunidad de estudiar varios aspectos de la normativa en cuestión (CENTENERA SÁNCHEZ-SECO, 2011, 237-268), y que consideramos modestamente que tal experiencia podría redundar en una aportación mayor de conocimientos para el alumnado.

${ }^{2}$ Confeccionado en base a lo que establece RODRÍGUEZ ESPINAR, 2009, 4. 
objetivos que pretendemos conseguir. A continuación, nos referimos a la metodología que hemos seguido a la hora de confeccionar la redacción de la norma anunciada. Seguidamente, ofrecemos un espacio dedicado a la valoración de la experiencia, dividido en dos partes. En la primera de ellas exponemos la percepción del alumnado sobre las actividades desarrolladas, teniendo en cuenta las respuestas que dio aquel a varias cuestiones de una encuesta. En la segunda analizamos los resultados obtenidos y planteamos algunos aspectos desde el punto de vista prospectivo.

Antes de comenzar con el desarrollo, quisiéramos dejar constancia de que no hemos pretendido que el alumnado elabore un trabajo de redacción normativa profesional, sino un trabajo académico de nivel básico. El problema de la violencia de género es complejo, y en una propuesta de modificación de la normativa actual debería adoptarse una perspectiva transversal que va más allá de aquellos ámbitos en los que pueda incidir la prevención, que es el campo que ha enmarcado el trabajo en cuestión. Incluso algunos de los aspectos abordados deberían haberse analizado de forma más detenida. Sin embargo, de un lado, para llevar a cabo un desarrollo de estas características hubiera sido necesario mucho más tiempo. La asignatura se cursa a lo largo de un cuatrimestre, en un horario de tres horas semanales, y su desarrollo requiere la realización de otras muchas prácticas (una muestra de ello puede verse en CENTENERA SÁNCHEZSECO, 2016a), además de las que aquí nos interesan. Por otra parte, conviene señalar que la mayor parte del alumnado matriculado está empezando el primer curso del Grado, y que un planteamiento transversal como el apuntado iría más allá del nivel de exigencia que debe considerarse en las circunstancias señaladas.

\section{El diseño de la experiencia}

Como señalamos en la introducción, las prácticas que presentamos vienen motivadas por la preocupante actualidad y persistencia de la violencia de género. No obstante, con aquellas tratamos también de aportar competencias en lo que respecta a una parcela que apenas se contempla en nuestro sistema docente, donde enseñamos a interpretar normas, pero no a crearlas. El esquema de trabajo en este ámbito es el que marcan las Directrices de técnica normativa que consideraremos de forma pormenorizada en los espacios siguientes. Al menos hasta donde alcanzamos a conocer, no tenemos constancia de experiencias en las que se haya tratado el problema que nos ocupa en el contexto docente, desde el ámbito de conocimiento señalado.

Más concretamente, en la experiencia que presentamos perseguimos los objetivos de la siguiente relación.

1. Formar en igualdad entre hombres y mujeres en la asignatura de Redacción de textos legales.

2. Fomentar en el alumnado el estudio, análisis y concienciación sobre el problema de la violencia de género. Concretamente, nos interesa referirnos al origen del problema y a sus diferentes manifestaciones. 
3. Aportar al alumnado conocimientos básicos sobre la normativa estatal que regula la cuestión.

4. Suscitar en el alumnado la formulación de propuestas de mejora normativa desde el punto de vista de la prevención.

5. Aportar conocimientos sobre la redacción de documentos administrativos y normas. En lo que respecta a estas últimas, centramos la atención en las disposiciones modificativas.

6. Fomentar el pensamiento crítico y reflexivo en base a los objetivos señalados anteriormente.

7. Fomentar el uso de los entornos virtuales, como la plataforma de la asignatura Blackboard e Internet.

Como puede apreciarse, la lista de objetivos es generosa. Su consecución requiere, por tanto, un planteamiento capaz de, al menos, vehicular acciones de diferente tipo necesarias para su consecución. Esta exigencia pensamos que queda satisfecha si recurrimos a aquellas tareas propias del proceso normativo que, sin duda, alcanzan a la redacción, pero van más allá (CENTENERA SÁNCHEZ-SECO, 2016b, 274-277). Como veremos en los siguientes espacios la complejidad de aquel ofrece cobertura suficiente para la consecución de los objetivos expuestos.

\section{El desarrollo metodológico}

\section{1. Planteamiento general}

Como podrá observarse a lo largo de los siguientes apartados, la metodología que seguimos a la hora de elaborar el trabajo de fin de curso no es lineal. Por ello, y con el fin de que el desarrollo siguiente pueda ser más claro, a continuación, presentamos un resumen general que da cuenta de la progresión llevada a cabo con las tareas realizadas. El ejercicio de redacción se entrega cuando termina el curso y hace las veces de examen final, en el caso de que se curse la asignatura conforme a la opción de evaluación continua. Sin embargo, la elaboración del trabajo se va desarrollando a lo largo de la asignatura. En las primeras semanas presentamos el problema que nos ocupará y dedicamos parte de algunas clases a comentar su origen, causas, tipos, etc. En esta primera etapa también llevamos a cabo una serie de actividades, en base a varios recursos, que pueden ayudar a redactar posteriormente la parte expositiva de la norma (nos referiremos a aquellas en el espacio dedicado a la parte en cuestión). Más adelante, hacia la mitad del curso, nos detenemos en las Directrices de técnica normativa. A medida que explicamos cada una de las partes de la norma siguiendo dicho documento, vamos realizando ejercicios que, en la medida de lo posible, tratamos de aprovechar para plantear un primer bosquejo de redacción del trabajo de fin de curso (principalmente, en lo que respecta al título y la parte expositiva). Como también precisaremos en lo que sigue, en las últimas semanas, una vez concluida la explicación de las Directrices de técnica normativa, publicamos en el aula virtual de la asignatura las pautas concretas para desarrollar el trabajo de fin de curso. En este momento, el alumnado debe llevar a cabo la redacción de las partes dispositiva y final de la norma, y 
también debe retomar las redacciones del título y de la parte expositiva (bosquejadas anteriormente, a medida que hemos explicado dichas partes), con el fin de completar aspectos que hayan podido quedar pendientes, mejorar la redacción y evitar posibles inconsistencias en el texto final.

Como ya adelantamos, se trata de un ejercicio individual, es decir, cada persona debe realizar su propio trabajo, ello con independencia de que puedan compartirse puntos de vista y determinados aspectos de las redacciones, a propósito de los ejercicios realizados en el aula. Por tanto, el rol principal que se adquiere es el de quien prepara textos legislativos desde un Ministerio. Secundariamente, hemos de señalar también que, como veremos más adelante, en lo que se refiere a la redacción de textos administrativos, colaterales al proceso legislativo, el alumnado trabaja como si formara parte del personal administrativo de un Ministerio, o bien de un grupo de investigación de una Universidad.

\section{2. Planteamiento concreto de cada parte de la norma}

\section{A. Título}

Las Directrices de técnica normativa establecen que el título de la norma debe incluir los siguientes aspectos: tipo de disposición, referencia a la modificación, título de la norma que se va a modificar ( partes en los que se van a llevar a cabo las modificaciones) y contenido sobre el que va a incidir la modificación (en nuestro caso, la prevención) (Resolución de 28 de julio..., 2005, 26880). Debe decirse, no obstante, que el título de la norma cambia cuando aquella se publica. En tal caso, si se trata de una disposición general debe figurar el tipo de disposición, el número de la ley seguido de barra y año, la fecha de promulgación, y después la parte esencial del título de la norma, que debe indicar su objeto, contenido o finalidad (SALVADOR CODERCH, 1989, 49). En nuestro caso, dado que pretendemos llevar a cabo una norma modificativa, la última parte del título debe ir precedida de la referencia a la modificación, y en ella debe figurar el título de la norma que se modifica y la materia en la que se incide. Con el fin de practicar en clase sobre estos aspectos, solicitamos al alumnado que realice varios ejercicios, entre ellos, los siguientes, que proponemos para comenzar a trabajar en la redacción del trabajo de fin de curso:

- "Redacta un título para la ley del trabajo de fin de curso, teniendo en cuenta que se trata de una norma que se va a enviar a las Cortes para su debate (título de norma no publicada)".

- "Redacta un título para la ley del trabajo de fin de curso, teniendo en cuenta que se trata de la primera norma publicada a comienzos del año próximo. En la fecha debe ponerse el día y mes en el que ha de entregarse el trabajo (título de norma ya publicada)".

Esta primera labor de redacción deberá estar presente en el trabajo de fin de curso. En los criterios que establecimos para su elaboración varias semanas después, señalamos 
que en lo que respecta al título de la norma, el alumnado debe redactar el que ha de tener la disposición desde que se comienza a elaborar, es decir, debe tratarse de un título de anteproyecto de ley ${ }^{3}$. Además, pedimos que en nota a pie de página, insertada al final de la redacción anterior, se escriba el título de la norma como si ya se hubiera publicado.

Aunque pueda parecer simple, la elaboración de estas redacciones entraña complejidad, no únicamente por los aspectos técnicos que han de tenerse en cuenta y que ya hemos señalado, sino también por las diferencias que deben observarse en cada caso. Además, debe decirse que en este punto la redacción conlleva un trabajo técnico adicional no exento de importancia, en lo que respecta al tipo de disposición. En esta cuestión incidimos cuando la asignatura estaba ya avanzada, tras haber explicado todas las partes de la norma y después de exponer las pautas para realizar el trabajo de fin de curso. En términos generales debe decirse que una ley ordinaria no puede regular materias reservadas a la orgánica (que es el rango de la norma que pretendemos modificar), en base al principio de competencia (ENÉRIZ OLAECHEA, 2007, 107, 108). Sin embargo, de la reserva de ley orgánica no se deriva la conclusión de que una materia ajena a aquella, por el hecho de recogerse en una ley orgánica, quede afectada por la "congelación de rango" (GARCÍA-ESCUDERO MÁRQUEZ, 2005). De este modo, puede darse el caso de que una ley orgánica aborde, a modo de desarrollo del núcleo de la materia orgánica o como complemento, materias que no se reservan a aquella. En tal caso, la modificación de estas últimas puede llevarse a cabo por medio de ley ordinaria (ENÉRIZ OLAECHEA, 2007, 108). La pregunta que se suscita en este punto es si los preceptos que vamos a modificar tienen rango orgánico o pueden incluirse en el supuesto recientemente expuesto. La respuesta debe buscarse en la propia ley orgánica que se pretende modificar, pues su texto indica los preceptos que se pueden modificar por ley ordinaria (GARCÍA-ESCUDERO MÁRQUEZ, 2005) ${ }^{4}$. Según las Directrices de técnica normativa que seguimos, hemos de localizar la respuesta en las disposiciones finales, donde queda establecida la naturaleza de la ley (Resolución de 28 de julio..., 2005, 26883). Concretamente, en el ejercicio que nos ocupa localizamos la información en cuestión en la disposición final tercera de la ley orgánica que vamos a modificar. De su lectura cabe deducir que, por ejemplo, el título preliminar, en el que incidirá parcialmente la parte dispositiva de la norma, como después veremos, tiene rango orgánico (Ley Orgánica 1/2004, 2015², 43). Por tanto, la norma modificativa que nos ocupa deberá ser ley orgánica.

\section{B. Parte expositiva}

\footnotetext{
3 Concretamente, desde el inicio del proceso el texto recibe el nombre de "anteproyecto de ley", y "proyecto de ley" cuando es aprobado por el Consejo de Gobierno para ser enviado al Parlamento. Sobre esta información NAVARRO ATIENZA, 2015, 80.

${ }^{4}$ Para corroborar tal argumento podemos citar como referencia jurisprudencial la Sentencia del Tribunal Constitucional 5/1981, fundamentos jurídicos 20 y 21.

${ }^{5}$ Ponemos el año de la última modificación de la ley orgánica.
} 
Casi desde el comienzo del curso, durante varias semanas vamos dedicando parte de algunas clases al análisis de la temática sobre la que versará el trabajo de fin de curso, siguiendo en este desarrollo las recomendaciones que encontramos en el ámbito de las políticas públicas, con vistas a abordar un problema determinado. La primera tarea a tener en cuenta en este sentido es definir el problema, algo que conlleva análisis relativos a sus posibles causas, sus consecuencias o su cuantificación (ZAPATERO, 2009, 84 y ss.). Con el fin de abordar estas cuestiones, vamos alojando en la plataforma virtual de la asignatura numerosos enlaces a documentos, dentro de una carpeta dedicada al trabajo de fin de curso. Se trata de referencias que debían leerse total o parcialmente antes de asistir a clase, con el fin de comentar su contenido en aquella. Concretamente, las referencias más relevantes fueron las siguientes ${ }^{6}$ :

- Constitución Española. 1978 (2011, 2, 3, 4), artículos 9.2, 14 y 15.

- Convención sobre la eliminación de todas las formas de discriminación contra la mujer. CEDAW. 1979 (2007, 10, 17-22).

- Convenio del Consejo de Europa sobre prevención y lucha contra la violencia contra las mujeres y la violencia doméstica $(2011,4,5)$.

- Informe de la Cuarta Conferencia Mundial sobre la Mujer. 1995 (1996, 51-52).

- Ley Orgánica 1/2004, de 28 de diciembre, de Medidas de Protección Integral contra la Violencia de Género. 2004 (2015, 1-9). Exposición de motivos y la primera parte dedicada a la prevención.

- Noticia de la Universidad de Alcalá. Día Internacional de la Eliminación de la Violencia contra la Mujer, una mirada a las nuevas generaciones (2017).

- Plan Nacional de Sensibilización y Prevención de la Violencia de género ([2009], 8, 9, 16-20).

- Víctimas mortales por violencia de género $(2017,1)$.

Con los recursos citados pretendemos que el alumnado adquiera conocimientos relativos a aspectos tales como el origen del problema, sus causas y consecuencias, la regulación estatal del mismo, las dimensiones que tiene actualmente o las recomendaciones procedentes del ámbito internacional. Con el fin de abundar en estos aspectos, en una de las sesiones contamos con la intervención de la profesora M. ${ }^{a}$ Remedios Menéndez Calvo, directora de la Unidad de Igualdad de la Universidad de Alcalá. En su participación incidió en aspectos tales como la importancia de las campañas de prevención, la atención en las nuevas tecnologías o la educación en todos los niveles en materias tales como la igualdad de oportunidades, la no discriminación por razón de sexo y el empoderamiento de las mujeres ${ }^{7}$.

\footnotetext{
${ }^{6}$ En las citas de normativa y documentación internacional hemos incluido el título de la referencia y la fecha en la que se publicó. A continuación, hemos puesto entre paréntesis la fecha de la última modificación del texto (en el caso de consultas de legislación consolidada), o de la publicación del trabajo que recoge la referencia citada, en su caso, seguida de las páginas.

${ }^{7} \mathrm{La}$ misma profesora, que forma parte también del proyecto de innovación docente citado al comienzo, recomendó la consulta de algunas de las referencias recogidas en la relación anterior, y aportó información al alumnado sobre eventos contra la violencia de género.
} 
Además de lo anterior, a lo largo del desarrollo de la asignatura solicitamos también al alumnado otras tareas que, más adelante, servirían para confeccionar la parte expositiva de la norma. Se trata de supuestos que alojamos en el aula virtual de la asignatura de forma paulatina, a partir de los cuales debía redactarse un documento administrativo. Concretamente, propusimos casos que podrían tener lugar en el proceso de producción normativa de la ley que debería redactarse finalmente. Además de formar en la redacción de documentos de tipo administrativo, con los ejercicios en cuestión también pretendimos que el alumnado trabajase con parte de la información recogida en varios de los documentos citados anteriormente. A continuación transcribimos los supuestos y los ejercicios de redacción solicitados a partir de aquellos:

"Supuesto. Imagina que trabajas en la Dirección General del Ministerio de Sanidad, Servicios Sociales e Igualdad. Estás preparando documentación para desarrollar un futuro borrador, con el fin de modificar la normativa estatal sobre medidas contra la violencia de género. Para ello, te vas a poner en contacto con el Observatorio Estatal de Violencia sobre la Mujer, para solicitar datos sobre el número de casos diagnosticados en los años más recientes, así como información sobre la evolución del problema. Por otra parte, también tienes interés en que te informen acerca de en qué ámbitos entienden que es mejor actuar.

Ejercicio de redacción. Redacta una carta de no más de una página, solicitando los datos señalados anteriormente".

"Supuesto. Imagina que trabajas como investigador/a en esta Universidad. Desde el Ministerio de Sanidad, Servicios Sociales e Igualdad se ponen en contacto contigo, pues tienen pensado modificar la normativa estatal de medidas contra la violencia de género. Más concretamente, te solicitan un informe en el que se resuman aspectos tales como el origen del problema, las posibles formas de violencia, las consecuencias del problema y las posibles medidas a adoptar. El texto debe redactarse teniendo en cuenta el Informe de la Cuarta Conferencia Mundial sobre la Mujer y La Convención sobre la eliminación de todas las formas de discriminación contra la mujer.

Ejercicio de redacción. Redacta un informe de no más de página y media, en el que desarrolles los contenidos señalados. Para llevar a cabo el planteamiento del texto puedes tener en cuenta la documentación de la carpeta dedicada al trabajo de fin de curso".

Una vez realizadas las tareas anteriores, y tras explicar la elaboración de la parte expositiva de la norma, de nuevo siguiendo las Directrices de técnica normativa, solicitamos al alumnado que preparara un primer borrador de aquella para el trabajo de fin de curso. A propósito de ello, recomendamos que se tuvieran en cuenta las tareas realizadas anteriormente, pues podrían ser de utilidad para desarrollar numerosos contenidos de la parte de la norma que nos ocupa. Concretamente, las directrices que seguimos establecen que en aquella debe dejarse constancia de los antecedentes de la 
cuestión, el objeto y finalidad de la norma, los informes solicitados ${ }^{8}$ y un resumen del contenido de aquella (Resolución de 28 de julio..., 2005, 26880, 26881). Como ya adelantamos, de igual forma que con el título, este primer borrador de la parte expositiva se tuvo que retomar en las últimas semanas de clase, una vez redactado el borrador completo de la norma.

\section{Parte dispositiva}

Como en los casos anteriores, a la hora de elaborar la parte que ahora nos ocupa también seguimos las recomendaciones de las Directrices de técnica normativa. Este documento desarrolla un itinerario para las disposiciones de tipo general. Sin embargo, la técnica es diferente si pretendemos afrontar la redacción de una disposición modificativa. En este caso se contemplan dos opciones, la modificación simple, cuando se pretende modificar una norma, y la múltiple, si el ejercicio en cuestión alcanza a diferentes disposiciones (Resolución de 28 de julio..., 2005, 26885). En nuestro caso, el modelo a tener en cuenta fue el primero, dado que la intención era modificar una única norma, la estatal sobre medidas contra la violencia de género. Lo recomendable en tal circunstancia es plantear un desarrollo conforme al siguiente esquema que expusimos en clase, a partir de lo que establece el documento que nos sirvió de guía (Resolución de 28 de julio..., 2005, 26885):

\section{(1) Artículo único con título que haga referencia a la disposición que se modifica}

(2) Texto marco general, que introduce las modificaciones

(3) Texto marco concreto (enumerado con cardinal de forma correlativa, a medida que se van añadiendo textos marco concretos), que solo hace referencia al texto que se modifica o se añade

(4) Texto modificado o añadido entre comillas

(3) $[\ldots]$

(4) $[\ldots]$

Cuadro 1. Esquema general de la parte dispositiva.

El planteamiento de las filas 3 y 4 del esquema se va repitiendo por cada modificación que se pretenda insertar en el texto normativo. Las modificaciones que debían quedar reflejadas en el trabajo las publicamos en el aula virtual de la asignatura, junto con el resto de criterios para elaborar el trabajo de fin de curso, y se basaron en las cuestiones consideradas en las sesiones anteriores, dedicadas al análisis del problema. En esta ocasión la lista recogió varias solicitudes, concretamente, dos de nueva redacción (la primera y la segunda de la siguiente relación) y cuatro de adición (el resto de solicitudes, siendo tres de adición de apartados y una de adición de artículo). Son las siguientes:

\footnotetext{
${ }^{8}$ El informe no se solicita en el caso de las leyes (Resolución de 28 de julio..., 2005, 26880). No obstante, pedimos que se incluyese una referencia al mismo, teniendo en cuenta que previamente habíamos realizado un ejercicio consistente en llevar a cabo un informe sobre la cuestión. 
- Modifica el artículo 1.1., redactando un concepto de violencia de género más amplio. A propósito de esta cuestión, consideramos el artículo 3 del Convenio del Consejo de Europa sobre prevención y lucha contra la violencia contra las mujeres y la violencia doméstica $(2011,4,5)$.

- Modifica el apartado 2 de artículo 3. Pon una palabra o expresión que solicite más compromiso que la palabra "impulsarán".

- Añade un apartado al artículo 3, a continuación del apartado 2, que garantice la continuidad de las campañas, que deben referirse a distintos tipos de violencia (física y psicológica), y desarrollarse en Internet y las redes sociales. El actual apartado 3 del artículo 3 deberá enumerarse con el número correcto.

- Añade un apartado al artículo 4, a continuación del apartado 7, que establezca la creación de una asignatura en todos los niveles educativos, que forme sobre igualdad de oportunidades, no discriminación por razón de género y empoderamiento de las mujeres.

- Añade un apartado al artículo 6 (que tendrá dos apartados que deberán enumerarse), que establezca que las Administraciones educativas darán ayudas para revisar los materiales existentes, crear otros nuevos, e investigar para seguir avanzando en el descubrimiento del papel que las mujeres han desempeñado en la historia.

- Añade un artículo a continuación del artículo 14, sobre los videojuegos, en el que se indique que la Administración pública debe promover acuerdos de autorregulación, para que las empresas se comprometan con la igualdad entre mujeres y hombres.

Una muestra de algunos puntos del texto final de la parte dispositiva, teniendo en cuenta las recomendaciones de las directrices, así como las solicitudes recientemente expuestas, podría ser la siguiente:

Artículo único. Modificación de la Ley Orgánica 1/2004, de 28 de diciembre, de Medidas de Protección Integral contra la Violencia de Género.

La Ley Orgánica 1/2004, de 28 de diciembre, de Medidas de Protección Integral contra la Violencia de Género, queda redactada como sigue:

[...]

Dos. El apartado 2 del artículo 3 queda redactado del siguiente modo:

"2. Los poderes públicos, en el marco de sus competencias, desarrollarán campañas de información y sensibilización específicas con el fin de prevenir la violencia de género".

$[\ldots]$

Cuatro. Se añade un apartado al artículo 4, a continuación del apartado 7, con la siguiente redacción:

"8. El sistema educativo incluirá una asignatura, adaptada a todos los niveles educativos, que forme sobre igualdad de oportunidades, no discriminación por razón de género y empoderamiento de las mujeres". $[\ldots]$ 
Seis. Se añade un artículo 14bis, que queda redactado como sigue:

"Artículo 14bis. Videojuegos.

La Administración pública promoverá acuerdos de autorregulación, para que las empresas que producen videojuegos se comprometan con la igualdad entre hombres y mujeres".

La elaboración de las modificaciones lleva consigo una serie de especificidades técnicas que deben tenerse en cuenta (así, en la última de ellas debía saberse qué tipo de enumeración ha de utilizarse a la hora de insertar un nuevo artículo). Además de ello, conviene subrayar que para realizar el ejercicio fue ineludible trabajar a partir del texto de la norma que se pretendía modificar. Ello propició que el alumnado adquiriese un conocimiento más preciso de varios preceptos de la normativa actual en materia de prevención, más allá del primer acercamiento que planteamos a la hora de presentar por primera vez la ley orgánica en la que nos íbamos a centrar.

\section{Parte final}

El trabajo concluye con la redacción de una serie de disposiciones que solicitamos también en el documento que recogía las pautas para realizar el ejercicio, tras haber considerado el contenido correspondiente de las Directrices de técnica normativa. Concretamente, los puntos que debía tener en cuenta el alumnado en esta ocasión son los siguientes:

- Redacta una disposición sobre el título competencial habilitante (puede servir de orientación buscar cómo se desarrolla esta cuestión en la Ley Orgánica 1/2004, o la Ley Orgánica 4/2007).

- Redacta una disposición que establezca una vacatio legis de 15 días.

- Redacta una disposición en la que se solicite a las Administraciones públicas un informe anual en el que se evalúen los efectos de las campañas realizadas.

- Redacta una disposición en la que se solicite a las Administraciones públicas una evaluación anual sobre la sensibilización de la población en materia de violencia de género, con el fin de proponer medidas en el futuro.

La redacción de esta parte de la norma entraña una complejidad técnica considerable. En esta ocasión debían redactarse los textos de las disposiciones, así como sus títulos. Estos últimos, según las Directrices de técnica normativa (Resolución de 28 de julio..., 2005: 26883), deben ir precedidos del tipo de disposición (adicional, transitoria, derogatoria o final, dependiendo del contenido de la disposición) y de su numeración, correlativa en cada tipo de disposición. La toma en consideración de estos aspectos técnicos recomienda que a la hora de abordar la parte final solicitada se siga el siguiente itinerario, que propusimos en el aula. Por una parte, conviene enmarcar cada una de las solicitudes anteriores en un tipo de disposición. Esta tarea debe llevarse a cabo teniendo en cuenta los contenidos de cada una de las disposiciones, anunciados en la relación anteriormente expuesta. El siguiente paso es ordenar las disposiciones obtenidas con la 
labor anterior, atendiendo al esquema que presentan las Directrices de técnica normativa, y a continuación enumerarlas. Así, por ejemplo, en las solicitudes propuestas hay dos disposiciones finales (la primera y la segunda), cuyos textos deberían ir al final de la parte que ahora nos ocupa, siguiendo el orden establecido en el documento que nos sirve de guía, con los siguientes encabezados, respectivamente: "Disposición final primera. Título competencial habilitante"; "Disposición final segunda. Vacatio legis".

\title{
4. Valoración de la experiencia
}

\section{1. Opinión del alumnado}

Cuando finalizó la asignatura solicitamos al alumnado que respondiese a una encuesta con cuatro cuestiones. Con ello pretendíamos obtener datos relativos al nivel de consecución de varios de los objetivos que nos propusimos en un principio. Fueron treinta y cinco personas las que respondieron a la encuesta. A continuación mostramos los resultados obtenidos en cada una de las cuestiones que planteamos.

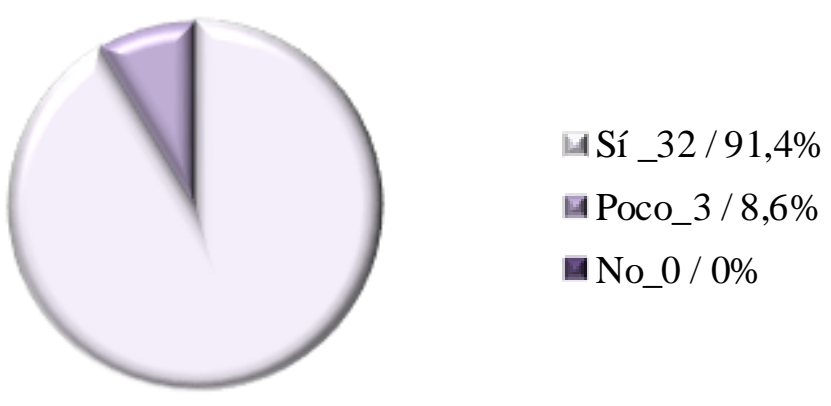

Gráfico 1. Resultados de la encuesta a la siguiente pregunta: ¿Te ha servido el trabajo de fin de curso para conocer la técnica legislativa de la norma modificativa?

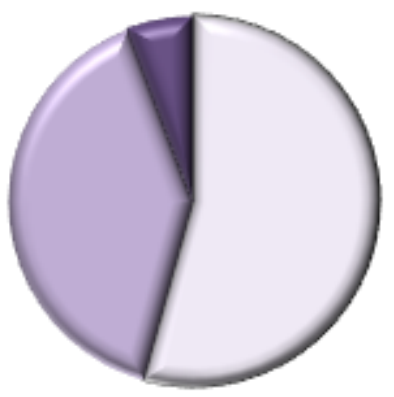

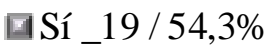 \\ 回 Poco_14 / 40\% \\ №_2 / 5,7\%
}

Gráfico 2. Resultados de la encuesta a la siguiente pregunta: ¿Habías oído hablar del origen del problema de la violencia de género, según lo hemos tratado en la asignatura, antes de cursar la misma? 


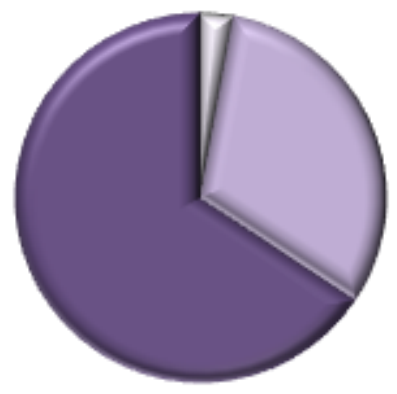

回Sí_1 / 2,9\%

回Poco_11 / 31,4\%

a No_23 / 65,7\%

Gráfico 3. Resultados de la encuesta a la siguiente pregunta: ¿Conocías la normativa estatal sobre medidas contra la violencia de género, antes de cursar la asignatura?

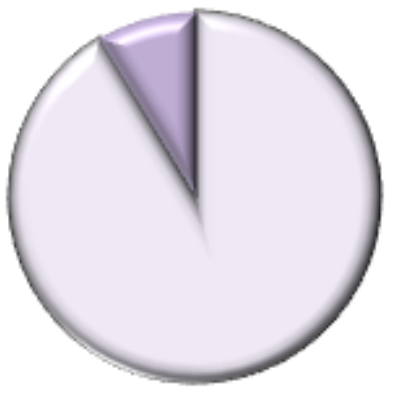

口í $32 / 91,4 \%$

回Poco_3 / 8,5\%

a No_0 / $0 \%$

Gráfico 4. Resultados de la encuesta a la siguiente pregunta: ¿Te ha parecido interesante el tema que hemos tratado en el trabajo de fin de curso?

En primer lugar, de los resultados expuestos llama la atención el alto porcentaje que se aprecia en dos respuestas afirmativas (el 91,4\% del alumnado), concretamente, las que observamos en las cuestiones 1 y 4 . En el primer caso, dicho resultado demuestra que buena parte del alumnado considera que el trabajo de fin de curso ha servido para adquirir conocimientos en lo que respecta a la técnica legislativa del tipo de normativa que nos ha ocupado. Del mismo modo, y si tenemos en cuenta las respuestas a la cuestión 4, podremos decir que en el mismo porcentaje los ejercicios realizados han reportado interés para el alumnado.

Por otra parte, resulta relevante constatar que más de la mitad del alumnado no conocía la normativa estatal sobre medidas contra la violencia de género (el 65,7\%), y que en un porcentaje menor (el 31,4\%) había oído hablar poco de aquella. Únicamente una persona conocía la ley orgánica modificada en el trabajo de fin de curso. Entendemos, por tanto, que la elaboración de este último puede ser una herramienta útil para acercar al alumnado al conocimiento de la normativa en cuestión. Para finalizar, creemos interesante constatar que las respuestas a la segunda cuestión descubren que más de la mitad del alumnado conocía el origen del problema (el 54,3\%), si bien es cierto que 
también en un porcentaje considerable (el 40\%), se había oído hablar poco del mismo, y que dos personas no habían oído hablar de él (el 5,7\%).

\section{2. Análisis de resultados y prospectiva}

Los ejercicios expuestos en este artículo, desarrollados en el ámbito de la redacción normativa y centrados en el tema de la violencia de género, demuestran que con ellos es posible contribuir a la adquisición de competencias en redacción, concretamente, teniendo en cuenta el planteamiento expuesto, en lo que respecta al desarrollo de disposiciones modificativas. La opinión del alumnado así lo certifica en un porcentaje muy importante. Varias de las actividades realizadas conllevan una complejidad considerable, quizá exigible en niveles posteriores. Por ello, aun cuando, como ya señalamos, comenzamos a abordar la temática del trabajo en las primeras semanas de curso, dejamos las dos últimas clases para que, en base al texto redactado hasta el momento, se expusiesen en el aula las dudas que hubieran podido surgir. Debemos subrayar que la asistencia a estas últimas sesiones fue bastante representativa y que las participaciones en aquellas fueron muy interesantes. No obstante, y aun siendo conscientes de las complejidades señaladas, pensamos que hemos de poner en valor la adquisición de los conocimientos que transmitimos con las labores realizadas al comienzo del Grado. Con ellos entendemos que proporcionamos al alumnado rudimentos básicos ineludibles a lo largo de sus estudios futuros. Parece evidente que quien se disponga a leer una norma -la cuenta de lecturas de este tipo se pierde a lo largo de los estudios jurídicos-, antes debe conocer sus partes y los pormenores de cada una de ellas. Sin embargo, al menos hasta donde sabemos, los conocimientos centrados en tales cuestiones no se aportan por lo general en los estudios del Grado en Derecho (incidimos en ello en CENTENERA SÁNCHEZ-SECO, 2016a, 147); más bien se dan por sabidos. En tal situación, parece casi obligado el aprendizaje autodidacta, pero la experiencia aquí expuesta demuestra que puede servir para atajar tal circunstancia, al menos en una parte considerable.

Por otro lado, y ya desde una perspectiva sustantiva, como ya señalamos la labor realizada se presenta también como posible recurso para plantear un primer acercamiento al conocimiento de la ley orgánica estatal de medidas contra la violencia de género, así como a aspectos tales como el origen del problema, sus causas o consecuencias. Ciertamente, en relación a esto último podría decirse que, según la encuesta realizada, más de la mitad del alumnado había oído hablar del origen del problema. Quizá ello se deba a que el mismo cada vez se esté haciendo más visible en ámbitos como el de la educación secundaria o los medios de comunicación. No obstante, y como ya señalamos, si de nuevo seguimos los resultados de la encuesta, hemos de señalar que en el resto de casos, en un porcentaje que se acerca a la mitad del alumnado, o bien se había oído hablar poco del problema (el 40\%), o bien nada (el $5,7 \%$ ). Ello sigue dando sentido al objetivo que consideramos en un principio, consistente en dar a conocer el origen del problema y las circunstancias del mismo. 
Por último, debemos destacar el interés que ha reportado para el alumnado la temática abordada (recordemos que así fue para el 91,4\% del mismo). En algún sentido -aunque no hasta los niveles alcanzados- previmos una respuesta afirmativa en esta cuestión durante el transcurso de la asignatura, pues las aportaciones del alumnado en clase en lo que respecta a, por ejemplo, cuestiones como el origen del problema, sus causas o consecuencias, fueron especialmente representativas. Ello, a nuestro modo de ver, puede deberse a varias razones. Por una parte, se trata de una cuestión, por desgracia, actual, cuya consideración en el aula pone encima de la mesa un problema persistente, sin resolver y que precisa solución. Por otra parte, entendemos que el interés quizá traiga también causa del desconocimiento existente acerca de los diferentes aspectos de la cuestión. En la labor que aquí hemos considerado tratamos aspectos sobre los que, al menos parte del alumnado, poco o nada conocía. Además, planteamos un acercamiento a la normativa estatal y trabajamos con numerosa documentación relacionada con la cuestión. Quizá el acceso a los contenidos que reporta todo este material, explique, al menos en parte, la respuesta que venimos considerando.

Desde un punto de vista prospectivo, habría que señalar, en base a lo dicho anteriormente, que la implementación futura de tareas como las que aquí hemos considerado puede resultar interesante, no únicamente por las habilidades y competencias que puedan transmitir desde la perspectiva técnica y en lo que respecta al contenido, sino también porque constatan en el ámbito académico un problema viejo pero tristemente actual. Su solución debe formar parte de la agenda parlamentaria, pero también de las de diferentes contextos ${ }^{9}$. En otro lugar señalamos que los nuevos planes de estudio ofrecen la cobertura necesaria para desarrollar aspectos de técnica legislativa en los trabajos de fin de Grado (CENTENERA SÁNCHEZ-SECO, 2016b, 284). Hemos tenido oportunidad de tutorizar ejercicios de este tipo en dicho ámbito, pero no a propósito del problema de la violencia de género. Quizá la experiencia aquí descrita y la adquisición de otros muchos conocimientos que proporciona el Grado en Derecho, hagan posible que, en algunos años, puedan aparecer propuestas ${ }^{10}$ para llevar a cabo proyectos en la línea planteada en este artículo, aunque con un mayor desarrollo, tanto en la extensión como en la transversalidad.

\section{Fuentes}

- CENTENERA SÁNCHEZ-SECO, F. (2011). ¿Podríamos haber tenido una ley de violencia de género de mayor calidad? Derechos y Libertades, 25, 237-268.

- CENTENERA SÁNCHEZ-SECO, F. (2016a). Recursos para formar en redacción de textos legales. Revista de Llengua i Dret, 65, 136-148.

\footnotetext{
${ }^{9}$ La idea está conectada con la solicitud de incorporar la perspectiva de género en la enseñanza del derecho. Sobre esto último, por ejemplo, PÉREZ-VILLALOBOS, 2014, 16.

${ }^{10}$ El alumnado puede realizar el trabajo de fin de Grado sobre un tema de su interés, conforme a la modalidad específica. Para ello, debe contar con el acuerdo de quien lleve a cabo la tutoría. Normativa para la presentación y defensa de los Trabajos Fin de Grado en la Facultad de Derecho. Universidad de Alcalá (2014), artículo 3.
} 
- CENTENERA SÁNCHEZ-SECO, F. (2016b). La formación en legística: aún una asignatura pendiente. Anuario da Facultade de Dereito da Universidade da Coruña, 20, 263-291.

- Convenio del Consejo de Europa sobre prevención y lucha contra la violencia contra las mujeres y la violencia doméstica (2011). Consultado en https://www.msssi.gob.es/ssi/igualdadOportunidades/internacional/consejoeu/CAHVIO. pdf. Fecha de consulta 21/03/2018.

- Constitución Española. 1978 (2015), Boletín Oficial del Estado, núm. 311, de 29 de diciembre de 1978. Consultado en http://www.boe.es/buscar/pdf/1978/BOE-A-197831229-consolidado.pdf. Fecha de consulta: 22/03/2018.

- Convención sobre la eliminación de todas las formas de discriminación contra la mujer. CEDAW. 1979 (2007). En: Convención sobre la eliminación de todas las formas de discriminación contra la mujer y el protocolo opcional a la Convención. S. 1.: Instituto de la Mujer. Ministerio de Trabajo y Asuntos Sociales. Consultado en http://www.csd.gob.es/csd/estaticos/myd/convencion_protocolo.pdf. Fecha de consulta 25/04/2018.

- ENÉREZ OLAECHEA, F. J. (2007). El Sistema de Fuentes del Derecho: de la Constitución Española al nuevo Derecho de la Unión Europea. Navarra: Thomson. Aranzadi.

- GARCÍA-ESCUDERO MÁRQUEZ, P. (2005). Sinopsis artículo 81. Consultado en http://www.congreso.es/consti/constitucion/indice/sinopsis/sinopsis.jsp?art=81\&tipo=2.

Fecha de consulta: 22/03/2018.

- Informe de la Cuarta Conferencia Mundial sobre la Mujer. Beijing, 4 a 15 de septiembre de 1995 (1996). Nueva York: Naciones Unidas. Consultado en http://www.un.org/womenwatch/daw/beijing/pdf/Beijing\%20full\%20report\%20S.pdf.

Fecha de consulta: 22/03/2018.

- Ley Orgánica 1/2004, de 28 de diciembre, de Medidas de Protección Integral contra la Violencia de Género. 2004 (2015). Boletín Oficial del Estado, núm. 313, de 29 de diciembre de 2004. Consultado en https://www.boe.es/buscar/pdf/2004/BOE-A-200421760-consolidado.pdf. Fecha de consulta: 05/04/2016.

- NAVARRO ATIENZA, M. A. (2015). Manual Práctico de Técnica Normativa. Sevilla: Instituto Andaluz de Administración Pública.

- Normativa para la presentación y defensa de los Trabajos Fin de Grado en la Facultad de Derecho. Universidad de Alcalá (2014). Consultado en http://derecho.uah.es/estudiantes/documentos/NUEVA_NORMATIVA_TFG.pdf. Fecha de consulta: 24/04/2018.

- PÉREZ-VILLALOBOS, M ${ }^{\mathrm{a}}$ C. (2014). La utilización de la metodología de género en la enseñanza-aprendizaje del Derecho Constitucional. Una experiencia en los primeros cursos del Grado en Derecho. Revista de Educación y Derecho, 9, 1-21.

- Plan Nacional de Sensibilización y Prevención de la Violencia de género ([2009]). S. 1.: Ministerio de Trabajo y Asuntos Sociales. Consultado en https://www.cop.es/GT/Plan_nacional_sensibilizacion_prevencion_violencia_genero.pd f. Fecha de consulta: 21/04/2018. 
- Redacción de textos legales (2017-2018). Consultado en https://www.uah.es/export/sites/uah/es/estudios/estudiosoficiales/grados/.galleries/Programas/G61/100076_G61_2017-18.pdf. Fecha de consulta 22/03/2018.

- Resolución de 28 de julio de 2005, de la Subsecretaría, por la que se da publicidad al Acuerdo del Consejo de Ministros, de 22 de julio de 2005, por el que se aprueban las Directrices de técnica normativa (2005). Boletín Oficial del Estado, núm. 180, de 29 de julio de 2005. Consultado en https://www.boe.es/boe/dias/2005/07/29/pdfs/A2687826890.pdf. Fecha de consulta 22/03/2018.

- RODRÍGUEZ ESPINAR, S. (2009). La investigación en docencia universitaria y los criterios de calidad en su difusión y reconocimiento. Revista de Educación y Derecho, $0,1-7$.

- SALVADOR CODERCH, P. (1989). El título de las leyes. En: Curso de técnica legislativa GRETEL. Madrid: Centro de Estudios Constitucionales.

- Sentencia del Tribunal Constitucional 5/1981, de 13 de febrero. Consultado en http://hj.tribunalconstitucional.es/es/Resolucion/Show/5. Fecha de consulta 22/03/2018.

- ZAPATERO, V. (2009). El Arte de Legislar. Pamplona: Thomson-Aranzadi.

- Víctimas mortales por violencia de género (2017). En: Ministerio de Sanidad, Servicios Sociales e Igualdad. Consultado en http://www.violenciagenero.msssi.gob.es/violenciaEnCifras/victimasMortales/fichaMuj eres/pdf/Vmortales_2017_04_12.pdf . Fecha de consulta: 12/12/2017 (esta estadística se va actualizando a lo largo del año. La versión que consultamos fue algo anterior a la de la fecha señalada). 